\title{
Cooperative and Non-Cooperative ARQ Protocols for Microwave Recharged Sensor Nodes
}

\author{
Marco Tacca, Paolo Monti, and Andrea Fumagalli \\ OpNeAR Lab, Erik Jonsson School of Engineering and Computer Science \\ University of Texas at Dallas \\ Richardson, TX 75080, USA \\ $\{$ mtacca,pxm017500,andreaf $\} @$ utdallas.edu
}

\begin{abstract}
The Generic Autonomous Platform for Sensor Systems, or GAP4S, is a maintenance-free wireless sensor network in which the sensor battery needs not be replaced. Power is delivered to the sensor via a microwave signal that is radiated by a base-station. The base-station also acts as the entry point to a wider communication network, e.g., the Internet.

This paper describes three automatic repeat request (ARQ) protocols that may be used in GAP4S to yield reliable and fair data transmission from the sensor nodes to the base-station. Two of the protocols take advantage of cooperative comnunication, whereby neighboring sensor nodes help during the retransmission process. The presented analysis on the saturation throughput of the ARQ protocols helps quantify the gain achievable when cooperative communication is used in GAP4S in a variety of working conditions.
\end{abstract}

\section{INTRODUCTION}

The advantages and potential impact of wireless networks are widely acknowledged nowadays. The commercial introduction of wireless LAN's, as well as the ongoing research activities in ad-hoc wireless networks and - more recently - wireless sensor networks, point at the widespread and increasing interest in these networks.

The deployment of sensor networks permits the distributed detection and estimation of various parameters related to a variety of commercial and military applications. Some applications include security, medical monitoring, machine diagnosis, chemical and biological detection [1]. Conventional sensor networks require wired links between the sensing element (sensor) and a central processing unit, where the signal processing takes place. In this case they are more commonly known as control networks [2]. It must be noted that the wiring

This research was supported in part by NSF Grants No. CNS0082085 , CNS-0435429 and ECS-0225528. process is often expensive, time-consuming, fault-prone, and potentially dangerous [3].

Both the recent development of relatively inexpensive and low-power wireless micro-sensors and various significant advances in wireless networking have paved the way to wireless sensor networks [4]-[13]. Some of the many benefits of wireless sensor networks include reduced installation cost, ability to rapidly reconfigure data acquisition, and safe deployment in inhospitable physical environments, such as disaster areas, toxic locations, and remote geographic regions. The wireless networking of the sensors allows them to organize themselves in order to jointly accomplish large sensing tasks, thus greatly improving the accuracy of the information provided to the user. For example, it is possible for a class of sensors to focus on particular aspects that are pointed out by another class of sensors, and to route their data to sink nodes or end-users outside the inspected area. In some instances, sensors are capable of establishing and maintaining their communication networks, and provide missing data by coordinating themselves to recover from failures. In summary, the sensor network may dynamically adapts itself to and interacts with the environment, sharing resources between the sensors and working in a power efficient way.

An interesting step forward in this field is represented by maintenance-free solutions, e.g, solutions where sensor or battery replacement is not required. Examples are the PicoRadio project at Berkeley and the $\mu$ AMPS (with base-station) at MIT. Both projects aim at short, or very short transmission distance $(2-10 \mathrm{~m})$, low cosi sensor nodes, and deployment of a large number of sensors, densely and uniformly distributed over the area of interest. At the sensor, the foreseen power dissipation level is below $100 \mu \mathrm{W}$ to avoid the use of batteries and permit energy-scavenging or harvesting [11] directly from the environment. To cope with the resulting short 
transmission range, ad-hoc multi-hop networking is envisioned among the highly dense sensors. In addition, the large number of sensors permits cooperative interactions for data fusion.

The Generic Autonomous Platform for Sensor Systems or GAP4S project [14] at the University of Texas at Dallas is in many respects complementary to the two above-mentioned efforts. It is indicated for those applications in which energy harvesting from the environment is neither possible, nor efficient, nor sufficient. The power consumption at the sensor node is above the harvesting level. Such a power level is provided by a (mini) base-station that remotely recharges the sensor on-board battery via a microwave (MW) signal. For the purpose of both recharging from and transmitting directly to the base-station, the sensor nodes in the GAP4S architecture must be inside the footprint of the base-station - possibly mobile - that represents the entry point to a wider communication network, e.g., the Internet. At any one time, the radius of the footprint may range up to hundred meters. Communication from the sensor to the base-station takes place on a radiofrequency (RF) channel. The power provided through the MW signal allows for relaxed requirement on $\mathrm{RF}$ accuracy on the channel from the sensor to the base-station. For example, a Voltage Controlled Oscillator (VCO) instead of a Phase-Locked Loop (PLLL) can be used at the sensor node. The MW signal generated by the base-station is also used to synchronize slots and to transmit acknowledgments and other control packets to the sensor nodes. The base-station may use smart antennas to ensure best power provisioning and full-duplex connectivity with the sensor nodes.

The objective of this paper is to propose and compare fair and reliable data protocols designed for the GAP4S architecture. Fairness is defined as the ability to give access at each sensor node to the channel bandwidth proportionately to its generated data rate. Reliable data delivery against transmission errors is accomplished by means of code redundancy and an automatic repeat request protocol (ARQ). The objective is accomplished by taking into account the effect of path loss on the MW recharging channel and both path loss and fading on the RF transmission channel. The main philosophy adopted here is to keep the sensor node as simple as possible. The base-station is responsible for scheduling collisionfree transmissions and -retransmissions of the sensor nodes, and guaranteeing fairness. For scheduling retransmissions, three ARQ protocols are considered, trading performance for implementation complexity at the base-station. The first is a conventional ARQ, whereby transmission from the sensor node to the base-station is repeated until successfully completed. The other two protocols take advantage of cooperative radio communication. In simple terms, cooperative communication is accomplished by requesting a node - other than the source -... to retransmit the data frame when the first transmission is not successful. Cooperative communication provides a way to borrow energy from other nodes to accomplish a successful data delivery. The next paragraph provides a short description of cooperative communication.

Radio networks are inherently different from wired ones, in that radio by its nature is a broadcast medium. When a node in the network transmits to a neighboring node, not only the destination node, but also other nodes within earshot receive the same signal. This phenomenon is conventionally treated as interference in the physical layer and -..- unless scheduling in time or frequency is provided - as collision in the MAC layer. Methods based on cooperative radio communication can turn this interference into an advantage [15]-[20]. Wireless nodes that are within earshot can cooperate by making use of the received interference and improve the overall capacity of the wireless links. The essence of the idea lies in that the destination benefits from data frames arriving via two statistically independent paths, a concept known as spatial diversity in wireless communications. It is believed that cooperative communication may bring several advantages to wireless networks in general, and it may become especially attractive for networks whose nodes have strict resource constraints, e.g., energy, such as sensor networks.

In the paper, a performance comparison is carried out among the three protocols, in terms of achievable saturation throughput. The comparison is achieved by varying a number of system parameters: transmission power, footprint size, path loss exponent, and antenna gain at the base-station. Results indicate that higher throughput, or equivalently lower power consumption for a target throughput is achievable by the two cooperative protocols, when compared to the conventional ARQ protocol.

\section{GAP4S DESCRIPTION}

This section is intended to provide the interested reader with a brief description of the unique nature of the GAP4S architecture.

Fig. 1 gives a pictorial description of the GAP4S architecture. The wireless sensor nodes are distributed 


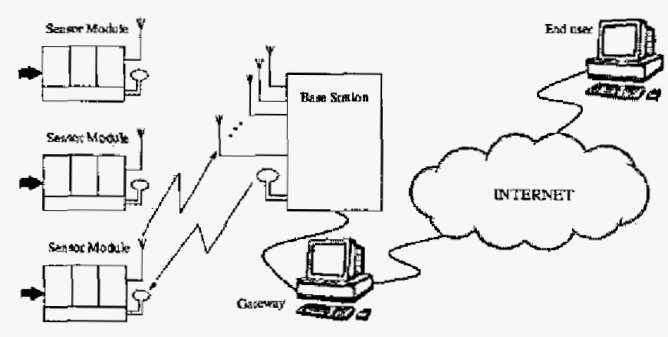

Fig. 1. GAP4S system architecture

on a fixed position. Their positions are geographically restricted to a predetermined area surrounding a powerrich mini base-station, i.e., the footprint size of the base-station. Each sensor node sends generated data to the base-station via a RF wireless uplink channel. Each sensor node recharges its battery with the received MW power that is continuously radiated by the basestation. A simple modulation, e.g., OOK, of the MW link enables data transfer from the base-station to the sensor nodes (downlink channel). The chosen RF data communication and data link protocol must ensure both reliable delivery of sensor data to the base-station and low-power consumption at the sensor nodes. The general philosophy followed to accomplish this double objective is to use dumb sensor nodes and provice all the network intelligence at the base-station. The sensor node and the base-station architectures are described next.

Fig. 2 shows the block diagram of the generic sensor node ${ }^{1}$. A variable number of sensors may be connected to the multi-functional sensor interface. The interface is capable of suitably handling signals coming from different sensor categories. Namely, the interface can process voltages, currents or capacitance and resistance modulations. Sensor electronic signals are then amplified by a digitally controlled gain factor. If any filtering action is required, the analog interface may host filters on demand. The $\mathrm{A} / \mathrm{D}$ converter is software programmable and permits to change the resolution from 8 to 12-bits and to adapt the conversion rate from low frequency up to $40 \mathrm{MHz}$. The power management module is integrated with the sensors, the analog interface and the data converter. The power management module controls the battery charge and discharge, determines the frequency of the main clock for an optimum power use, incorporates the $\mathrm{AC}-\mathrm{DC}$ converter for recharging

'An integrated prototype of the sensor node is currently under development [2] $],[22]$.

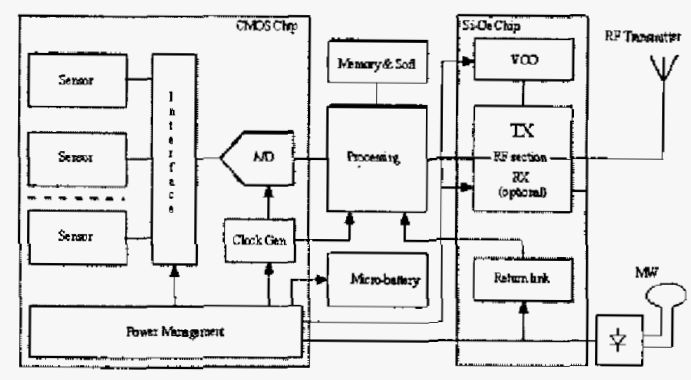

Fig. 2. GAP4S sensor node: block diagram

the battery (using the MW signal from the base-station) and manages the RF module. The micro-battery is currently a commercially available component. The power management module operates its functions on the basis of expected charge-discharge operative conditions. The signal processing and memory modules are based on existing low-power commercial component. The VCO RF modules are integrated using a sub-micron Si-Ge BiCMOS technology. The return link module recovers the slot synchronism and extracts messages transmitted by the base-station over the MW channel. An optional module may be added to the sensor node to provide the RF reception capability that is required by two of the data link protocols discussed in Section III.

The base-station receives data from the sensor nodes on the RF channel. A directional antenna may be used at the base-station to improve the received signal to noise ratio (SNR). Continuously, the base-station radiates recharging power to the sensor nodes using the MW channel. The radiated recharge power is constrained to safety levels. The MW signal is modulated to distribute slot synchronization, poll the sensor nodes for collision free uplink transmission, send ACK/NAK for received data frames, download software updates, and remotely program sensor nodes for the desired sensing operation. Unlike other solutions, downlink transmission is not costly to the sensor nodes as it occurs over the MW recharging channel. The base-station is also responsible for a collision free, reliable and fair collection of data from the entire set of sensor nodes, despite of their location. For this purpose it is necessary to design a data link protocol that makes the RF channel reliable and equally available to the sensor nodes.

\section{THREE ARQ PROTOCOLS FOR GAP4S}

This section describes three Automatic Repeat Request (ARQ) protocols that may be used in the GAP4S 
to overcome transmission errors. It is assumed that transmission errors may occur only on the uplink RF channel, as the sensor node power budget limits the SNR. Transmission errors on the downlink MW channel are negligible due to the relatively high power of the MW signal and can be easily overcome with a timeouttriggered retransmission at the base-station.

The three ARQ protocols take into account the unique nature of the GAP4S architecture, whereby the basestation - characterized by non-stringent power constraints - remotely recharges the sensor nodes, broadcasts slot synchronization, polls sensor nodes for collision free uplink transmissions, requests uplink data frame transmissions and retransmissions, and sends acknowledgments. All decisions are made at the basestation. Sensor nodes obey to the control frames received

from the base-station. The base-station is responsible for the choice of the cooperating sensor nodes and for scheduling collision-free transmissions and retransmissions of the sensor nodes.

\section{A. ARQ-NC Protocol}

The ARQ-NC is a non cooperative protocol, i.e., a conventional ARQ protocol. Upon request from the basestation, the sensor node transmits its data directly to the base-station (single hop communication). The data frame contains data encoded using an error detection and correction code. Upon the reception of a data frame either with errors that can be corrected, or no detected errors, the base-station replies with a positive ACK frame. Upon reception of a frame with detected errors that cannot be corrected, the base-station sends a NAK frame to the sensor node. In turn, the sensor node retransmits the data frame until reception at the basestation is successful. Available options for this protocol are stop and wait, go back N, selective repeat, etc. [23].

\section{B. ARQ-C Protocol}

The ARQ-C protocol takes advantage of the broadcast nature of the sensor node transmission by using spatial diversity to reach the base-station. Fig. 3 sketches how the ARQ-C protocol works. When the data frame transmitted by the sensor node (the source) is not successfully received, the base-station requests the frame retransmission by means of a second sensor node (the relay). The relay may have overheard ${ }^{2}$ the transmission of the source data frame, and stored the frame temporarily. If chosen wisely, the relay may increase the probability of

${ }^{2}$ In this case, the RF reception module is required at sensor nodes.

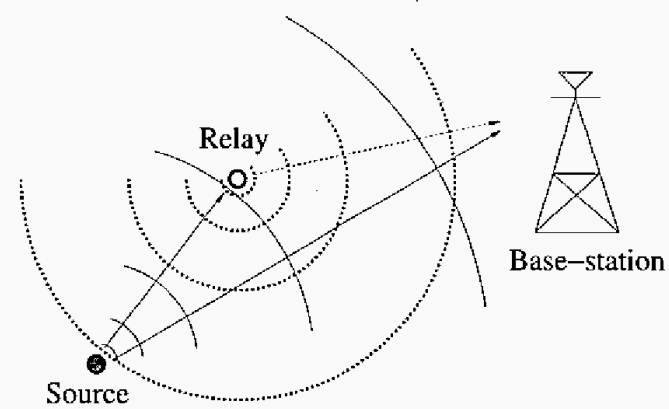

Fig. 3. Cooperation between two sensor nodes

delivering the data frame successfully without requiring any further retransmission and/or require a lower power to transmit the data frame to the base-station.

In the ARQ-C simplest version, the base-station broadcasts the following information: the identification of the source node that transmits next, the data frame to be transmitted, and the identification of the node that acts as the relay for this data transmission. If the data frame transmission from the source node to the base-station is successful, the base-station begins a new transmission cycle. If the transmission is unsuccessful, the relay node is called in to help with the frame retransmission. If the data frame transmission from the relay to the base-station is successful, the base-station begins a new transmission cycle. The relay node can help only if it has correctly received the data frame transmitted by the source. If the base-station does not hear from the relay, it safely assumes that transmission of the data frame from the source to the relay was not successful. It is also possible that the relay retransmission attempt is unsuccessful. Under either circumstances, the base-station requires retransmission of the data frame to the source and repeats the cycle.

The relay is viewed as a cooperating node in the effort of delivering the source data frame to the base-station. The cooperating node offers both space diversity and its own power budget. The ARQ-C protocol in GAP4S can select among multiple potential cooperating nodes to help the same source node. Assume that the density of the sensor nodes is high. It is likely that a number of sensor nodes may act as cooperating nodes for the same source.

For each transmission attempt, only one of these sensor nodes is chosen to be the relay. The base;station makes such choice, thus effectively creating a situation of load (and power) balancing among the sensor nodes. 
The base-station may choose in a probabilistic way according to some predefined distribution values. Note that the required intelligence is entirely residing at the base-station. Sensor nodes are ordered to overhear and transmit by the base-station via the recharging $\mathrm{MW}$ channel.

The above solution is not to be confused with the conventional store and forward solution. In fact, the latter is a layer 3 solution that requires routing tables at the sensor nodes. The former is a layer 2 solution in which the base-station is the centralized controller, allowed to chose cooperating nodes at each transmission attempt.

\section{ARQ-C $C^{N}$ Protocol}

The ARQ-C ${ }^{N}$ protocol is a recursive version of the ARQ-C protocol. Fig, 4 sketches how the ARQ-C ${ }^{N}$ protocol works. The source transmits to the base-station. Relay $i$ overhears ${ }^{3}$ and stores the data frame. If the source data frame is not correctly received, the basestation requires relay $i$ to retransmits the frame. (If relay $i$ does not transmit, the base-station can safely assume that relay $i$ did not receive correctly from the source and requires the source to retransmit again.) During the retransmissions at relay $i$, relay $j$, chosen by the basestation, overhears and stores the data frame. If the data frame retransmission from sensor node $i$ is not correctly received, the base-station requires relay $j$ to retransmits the frame. (If relay $j$ does not transmit, the base-station can safely assume that relay $j$ did not receive correctly from relay $i$ and requires relay $i$ to retransmit again.) This procedure continues recursively, possibly requiring the cooperation of additional relay nodes, until the frame is correctly received at the base-station.

The rationale behind this recursive protocol is the assumption that the relay node is chosen to have (a) a higher probability of successful (re)transmission than the one of the source (or previous relay) and/or (b) to require a lower power to transmit the data frame to the basestation. Thus, when retransmission is required, the data frame migrates to sensor nodes that are more likely to produce a successful transmission. For each data frame transmission, the sequence of the relay nodes is chosen by the base-station. Once again, the base-station may choose that in a probabilistic way, according to some predefined distribution values. The complexity at the base-station in this case is slightly higher than the one of the ARQ-C protocol, as the migration of each data frame must be tracked. If necessary, in order reception

${ }^{3}$ The base-station chooses node $i$ as the relay.

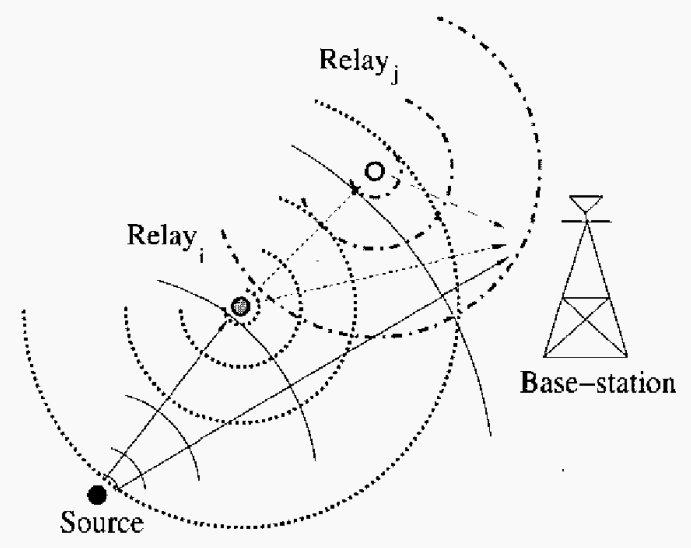

Fig. 4. Collaborations among three sensor nodes

of frames may be enforced by the base-station. In this protocol too, the required intelligence is entirely residing at the base-station. Sensor nodes are ordered to overhear and transmit by the base-station via the recharging $\mathrm{MW}$ channel.

A final note on the similarities and differences between the ARQ-C $C^{N}$ protocol and the store and forward. The similar aspect is that the data frame may reach the base-station via a multi-hop route. The difference is that the $\mathrm{ARQ}-\mathrm{C}^{N}$ remains a layer 2 protocol, in which the base-station acts as a central controller, while store and forward is a layer 3 solution based on routing tables stored at the sensor nodes.

\section{Assessing Saturation Throughput of the ARQ PROTOCOLS}

This section attempts to assess the saturation throughput that is achieved by the three retransmission protocols discussed in the previous section. The saturation throughput $(S)$ defines the throughput values $(\leq S)$ that can be sustained by the system under two constraints: the average power consumption at each sensor node cannot exceed the power recharge rate, and fair access is granted to all sensor nodes in the area surrounding the basestation. It is assumed that other system aspects are not limiting the system throughput, e.g., the channel and electronics bandwidth, buffer capacity, network latency, QoS, etc.

The sensor nodes and the base-station are stationary. The battery recharge characteristic at each sensor node is ideal, i.e., linear and indefinitely rechargeable. The relative data traffic intensity from each sensor node is known. 


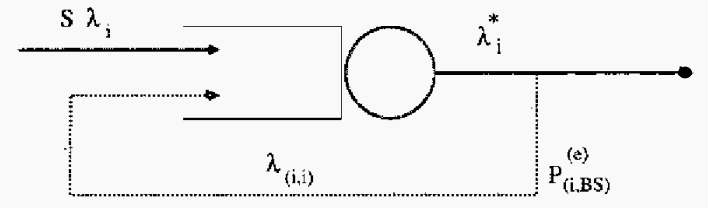

Fig. 5. ARQ-NC: flow model

The base-station determines which are the cooperating nodes for each source (ARQ-C and ARQ-C ${ }^{N}$ protocols only). The cooperating nodes are chosen to maximize the saturation throughput, as explained next.

The problem of maximizing the throughput with the three different protocols can be formulated using a flow model that relies on the following input and variables. Input:

- $N$ : number of sensor nodes,

- $P_{B S}$ : recharge power radiated on the MW channel by the base-station,

- $P_{i}^{r e c}$ : amount of recharge powér radiated by the base-station that reaches sensor node $i$,

- $d_{i, j}$ : distance between sensor node $i$ and $j$,

- $d_{i, B S}$ : distance between sensor node $i$ and the basestation,

- $E_{b_{i}}$ : energy transmitted per bit at sensor node $i$,

- $E_{b}^{(R x)}$ : energy necessary to receive one bit when a sensor node acts as the relay,

- L: number of bits per data frame,

- $P_{(i, j)}^{(e)}$ : data frame error probability from sensor node $i$ to $j$,

- $P_{(i, B S)}^{(e)}$ : data frame error probability from sensor node $i$ to the base-station.

\section{Variables:}

- $S \cdot \lambda_{i}$ : traffic intensity generated at sensor node $i . S$ is a real valued variable, i.e., $S \in \Re$. $\lambda_{i}$, measured in data frames per seconds, is a predefined constant that defines, per each sensor node, the flow of new data frames ${ }^{4}$.

\section{A. ARQ-NC Protocol}

Fig. 5 shows the flow model used for the ARQ-NC protocol. The figure represents the transmission queue at sensor node $i$. With probability $P_{(i, B S)}^{(e)}$ the data frame transmission is not successful, in which case the frame remains in the transmission queue for the next retransmission attempt. $\lambda_{i}^{*}$ is the relative total flow of frames

\footnotetext{
${ }^{4}$ This definition of the traffic intensity at each sensor node $i$ permits to model the traffic intensity at every node with a single variable, i.e, $S$.
}

transmitted by sensor node $i$, i.e., both transmissions and retransmissions. The problem of maximizing the throughput can be formulated as follows:

$$
\max : S
$$

Subject to:

$$
\begin{gathered}
\left(\lambda_{i}^{*} \cdot L \cdot E_{b_{i}}\right) \leq P_{i}^{r e c} \quad, \quad \forall i \in N \\
\lambda_{i}^{*}=\frac{S \cdot \lambda_{i}}{1-P_{(i, B S)}^{(e)}} \quad \forall i \in N
\end{gathered}
$$

Equation (2) ensures that the received recharge power at each sensor node is sufficient to sustain the total number of transmissions. Equation (3) expresses the total number of transmissions per unit of time.

\section{B. ARQ-C Protocol}

Fig. 6 shows the flow model used for the ARQC protocol. In this model multiple queues are used at each sensor node. Each sensor node $i$ has a separate queue to deal with data frames that correspond to the situation where sensor node $i$ is the relay for another node, including the case when sensor node $i$ is being the relay for itself, i.e., the base-station requires sensor node $i$ to retransmit its own packet ${ }^{5}$.

After a transmission error at sensor node $i$, flow $\lambda_{i}^{*}$ is divided into all the possible relay nodes, i.e., $\lambda_{(i, j)}$ represents the amount of data frames that suffered an error and requires (through the centralized control at the base-station). sensor node $j$ to be the relay. $\lambda_{(i, j)}$ is measured in data frames per second. The probability, $p_{(i, j)}$, that the base-station chooses node $j$ to be the relay for sensor node $i$ is then, $p_{(i, j)}=\frac{\lambda(i, j)}{\lambda_{i}^{*} P_{i, B S}^{(e)}}$. Notice that the solution of the presented LP model can be therefore used to set the values of the probabilities $p_{(i, j)}$ at the base-station.

Obviously, sensor node $j$ can be the relay only if the transmission from sensor node $i$ to sensor node $j$ is error free. $\bar{\lambda}_{(i, j)}=\left(1-P_{(i, j)}^{(e)}\right) \lambda_{(i, j)}$ is the flow of data frames - from $\lambda_{(i, j)}$ - successfully reaching sensor node $j$ from sensor node $i$. When calculating the power dissipated to overhear data frame transmissions at node $j$, i.e., when sensor node $j$ is acting as the relay node of node $i$, it is not possible to know a-priori whether data frames form source node $i$ are affected by

\footnotetext{
${ }^{5}$ Notice that having separate queues is helpful to describe the model but it is not strictly necessary in the actual implementation of the protocol.
} 


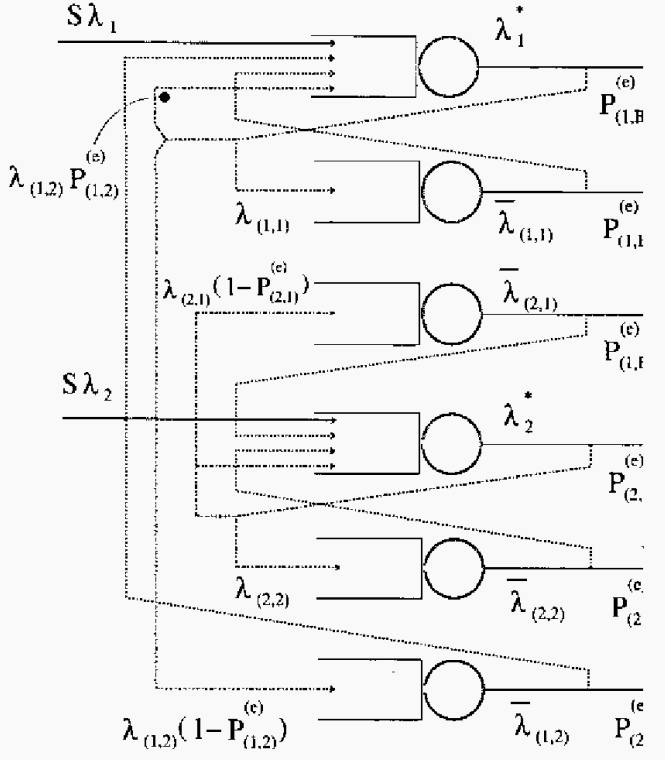

Fig. 6. ARQ-C: flow model

a transmission error or not. When node $j$ is selected as a relay, it has therefore to overhear all frames transmitted per unit of time at node $i$. The total number of data frames transmitted per unit of time at sensor node $i$ is $\lambda_{i}^{*}+\sum_{j} \bar{\lambda}_{(j, i)}$. The number of frames per unit of time relay node $j$ has to overhear from sensor node $i$

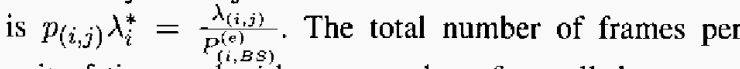
unit of time node $j$ has to overhear from all the sensor nodes is therefore $\sum_{i, i \neq j} \frac{\lambda_{(i, j)}}{P_{(i, n, s)}^{(e)}}$.

The problem of maximizing the throughput can be formulated as follows:

$$
\max : S
$$

Subject to:

$$
\begin{gathered}
\left(\sum_{j, j \neq i} \frac{\lambda_{(j, i)}}{P_{(j, B S)}^{(e)}}\right) \cdot L \cdot E_{b}^{(R x)}+ \\
+\left(\lambda_{i}^{*}+\sum_{j} \bar{\lambda}_{(j, i)}\right) \cdot L \cdot E_{b_{i}} \leq P_{i}^{r e c} \quad \forall i \in N \\
\lambda_{i}^{*}=S \cdot \lambda_{i}+\sum_{j}\left(P_{(i, j)}^{(e)} \cdot \lambda_{(i, j)}\right)+ \\
+\sum_{j}\left(P_{(j, B S)}^{(e)} \cdot \bar{\lambda}_{(i, j)}\right) \quad \forall i \in N
\end{gathered}
$$

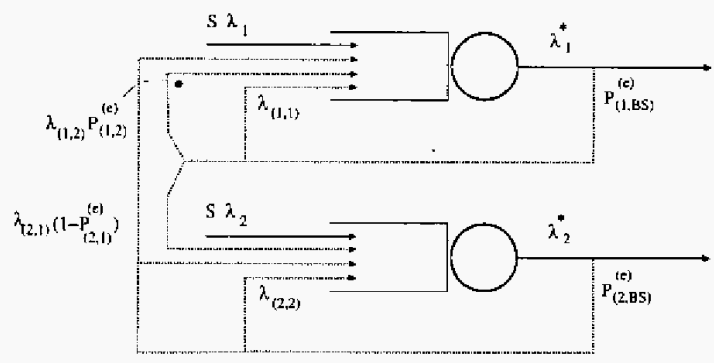

Fig. 7. ARQ-C $\mathrm{C}^{N}$ : flow model

$$
\begin{array}{cc}
\bar{\lambda}_{(i, j)}=\left(1-P_{(i, j)}^{(e)}\right) \cdot \lambda_{(i, j)} & \forall i, j \in N \\
\sum_{j} \lambda_{(i, j)}=P_{(i, B S)}^{(e)} \cdot \lambda_{i}^{*} \quad \forall i \in N
\end{array}
$$

Equation (5) balances the total energy used to transmit and overhear data frames and the energy received from the base-station. Equation (6) expresses the total number of data frames that sensor node $i$ has to transmit/retransmit. This is the sum of three terms: new data frames, data frames that the base-station designated to be retransmitted by relay $j$ but were not successfully received at sensor node $j$, and data frames that node $i$ has to retransmit because the retransmission operated by relay $j$ was not successful. Equations (7) and (8) are flow conservation constraints. Equation (8) ensures that $\lambda_{(i, j)} \leq P_{(i, B S)}^{(e)} \cdot \lambda_{i}^{*}$, therefore it always is $p_{(i, j)} \in[0,1]$.

\section{C. $A R Q-C^{N}$ Protocol}

Fig. 7 shows the flow model used for the ARQ-C ${ }^{N}$ protocol. Since at each retransmission the relay can take further advantage of another relay, only one queue at each sensor node is needed in this model. Equations for this protocol are similar to the previous case.

$$
\max : S
$$

Subject to:

$$
\begin{gathered}
\left(\sum_{j, j \neq i} \frac{\lambda_{(j, i)}}{P_{(j, B S)}^{(e)}}\right) \cdot L \cdot E_{b}^{(R x)}+ \\
+\left(\lambda_{i}^{*} \cdot L \cdot E_{b_{i}}\right) \leq P_{i}^{r e c} \quad \forall i \in N \\
\lambda_{i}^{*}=S \cdot \lambda_{i}+\sum_{j}\left(P_{(i, j)}^{(e)} \cdot \lambda_{(i, j)}\right)+ \\
+\sum_{j}\left(\left(1-P_{(j, i)}^{(e)}\right) \cdot \lambda_{(j, i)}\right) \quad \forall i \in N
\end{gathered}
$$




$$
\sum_{j} \lambda_{(i, j)}=P_{(i, B S)}^{(e)} \cdot \lambda_{i}^{*} \quad \forall i \in N
$$

Equation (10) balances the total energy used to transmit and overhear data frames and the energy received from the base-station. Equation (11) expresses the total number of frames that sensor node $i$ has to transmit/retransmit. This is the sum of three terms: new data frames, data frames that the base-station designated to be retransmitted by relay sensor node $j$ but were not successfully received at sensor node $j$, and frames that relay $i$ has to retransmit because the retransmission operated by relay $j$ was not successful. Equation (12) is a flow conservation constraint.

\section{PERformance}

This section reports various saturation throughput results achievable by the three retransmission protocols in a number of anticipated GAP4S scenarios. Results are presented after a short description of the assumptions made on the wireless channel.

\section{A. Wireless Channel Assumptions}

Both path loss and fading are taken into account in the RF uplink transmission. Only path loss is taken into account in the MW downlink recharging signal.

The path loss is modeled as

$$
E_{b_{r}}=E_{b_{t}} \cdot \frac{G_{T} \cdot G_{R}}{(4 \pi-d / \lambda)^{n}}
$$

where:

- $E_{b_{r}}, E_{b_{t}}$; energy per bit at the receiver and transmitter, respectively,

- $G_{T}, G_{R}$ : transmitter and receiver antenna gain, respectively,

- $d$ : distance between the transmitter and the receiver,

- $\lambda$ : wavelength at the channel center frequency,

- $n$ : path loss exponent, $n=2$ in free space, typically $2 \leq n \leq 4$ for environments with structures and obstacles [24], [25].

Fading is assumed to be Rayleigh slow and flat; i.e., the fading coefficients are considered constant over a single frame transmission. The fading experienced by each frame transmission is statistically independent of the fading experienced by any other frame transmission. The instantaneous signal to noise ratio of the RF channel at receiver $j$ given a transmission from transmitter $i$ is:

$$
\gamma_{(i, j)}=\frac{E_{b_{r}}}{N_{0}} \cdot \alpha_{(i, j)}^{2}
$$

where:
- $E_{b_{r}}$ : energy per bit at the receiver, calculated using (13),

- $N_{0}$ : noise spectral density of the Additive White Gaussian Noise (AWGN), proportional to the Boltzmann constant and the absolute temperature,

- $\alpha_{(i, j)}$ : a Raylejgh distributed random variable used to model the Rayleigh fading magnitude between transmitter $i$ and receiver $j, E\left[\alpha_{(i, j)}^{2}\right]=1 \forall i, j$.

It is assumed that the MW downlink channel is error free. On the RF uplink channel, sensors send data, augmented with a cyclic redundancy (CRC) code. Each block contains $B$ bits (including the CRC bits). The probability of receiving a frame incorrectly (error probability) is a function of both $\gamma_{(i, j)}$ and the code (if any) used to add redundancy to the transmitted data. The probability of detecting an erroneous codeword $P_{(i j)}^{(\text {block })}$ when a coded data frame, i.e. a codeword, is sent from transmitter $i$ to receiver $j$ is upper bounded by the following expression [17], [26]

$$
\begin{array}{r}
\zeta=\min \left\{1, \sum_{D=D_{f}}^{\infty} a(D) \cdot P\left(D \mid \gamma_{(i, j)}\right)\right\} \\
P_{(i, j)}^{(b l o c k)} \leq 1-\int_{0}^{\infty}(1-\zeta)^{B} \cdot p\left(\gamma_{(i, j)}\right) d \gamma_{(i, j)}
\end{array}
$$

where:

- $B$ : number of data bits in each block (data plus $\mathrm{CRC}$ bits), i.e., number of trellis branches in the codeword,

- $D_{f}$ : free distance of the code [27],

- $a(D)$ : spectrum of the code [28], i.e., number of codewords of weight $D$,

- $P\left(D \mid \gamma_{(i, j)}\right)$ : probability that a wrong path at distance $D$ is selected,

- $p\left(\gamma_{(i, j)}\right)$ : probability density function of the instantaneous SNR.

It is assumed that binary PSK with soft decoding is employed, in which case

$$
P\left(D \mid \gamma_{(i, j)}\right)=Q\left(\sqrt{2 \cdot D \cdot \gamma_{(i, j)}}\right)
$$

where $Q(\cdot)$ is the Marcum Q function [29] and $D$ is the weight of the codeword.

The CRC is used to detect the case of an erroneous decoding of a codeword, in which case a retransmission is requested. We assume that the $\mathrm{CRC}$ is able to detect all erroneous codewords, therefore the data frame error probability, i.e., the probability that a retransmission is requested is $P_{(i, j)}^{(e)}=P_{(i, j)}^{(b l o c k)}$. 


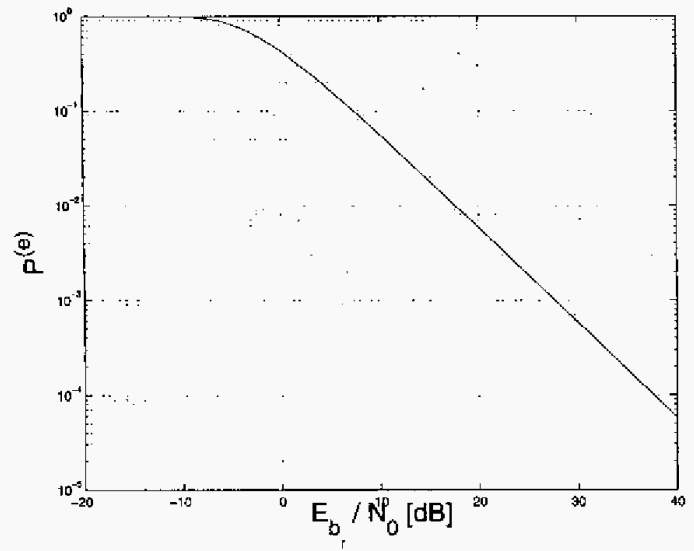

Fig. 8. Error probability $\left(P^{(e)}\right)$ as a function of $E_{b_{r}} / N_{0}$

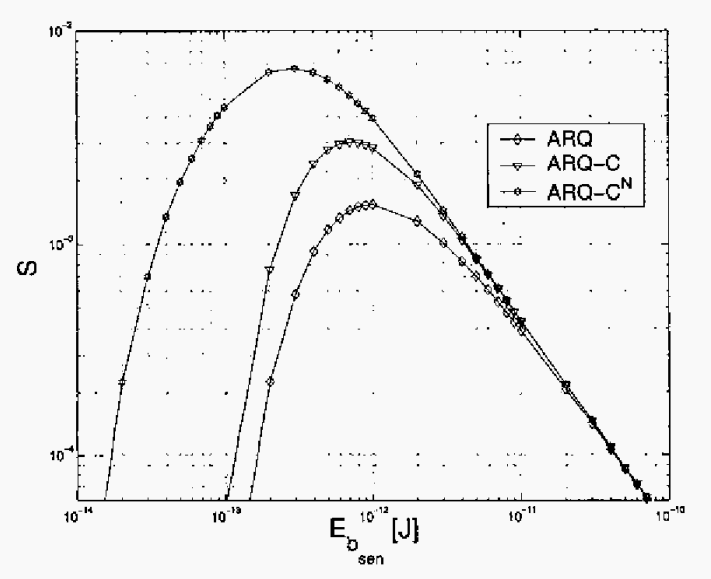

Fig. 9. Scenario A: saturation throughput $(S)$ versus energy per bit transmitted at the sensor nodes $\left(E_{b_{\text {as: }}}\right) . R=50 \mathrm{~m}, G=50$, $n=3.5$

\section{B. Numerical Results}

Numerical results are obtained using the GAP4S frequencies, i.e., $433 \mathrm{MHz}$ for the uplink and $2.4 \mathrm{GHz}$ for the downlink. Data frames have fixed length and carry $B=128$ bits (data plus CRC), that are encoded into 256 bit codewords using a rate-compatible punctured convolutional code (RCPC) with rate $1 / 2$, parent code rate of $1 / 4$, puncturing period of 8 , and memory of 4 [28]. The frame error probability $P^{(e)}$ versus $E_{b_{r}} / N_{0}$ for this RCPC is shown in Fig. 8. The recharge power that is constantly radiated by the base-station is $P_{B S}=10 \mathrm{~W}$. It is assumed that the energy received by the sensor antenna is fully transferred into its battery, and circuitry losses are negligible. It is assumed that the energy consumption at the sensor node is due to transmissions. It is assumed that the energy per bit necessary to overhear a transmission is negligible, i.e., $E_{b}^{(R x)}=0 \mathrm{~J}$. The consumption of the other sensor sections, e.g., analog-digital conversion, processing, power management, receiver, is neglected. Traffic is uniform, i.e., $\lambda_{i}=1, \forall i \in N$.

Saturation throughput is computed by solving the formulations presented in Section IV using ILOG Cplex [30]. Two distributions of the transmission power levels at the sensor nodes are considered:

- scenario A: the transmission energy per bit $E_{b_{s e n}}$ is the same at each sensor node

- scenario B: the transmission energy per bit at each sensor node is set to yield the same time-average signal to noise ratio $S N R_{B S}$ at the base-station.

Average values are computed over 20 distinct instances of sensor node distribution. Each instance is obtained by randomly distributing 200 sensor nodes within a circular footprint of radius $R$. The base-station is at the center of the footprint. The polar coordinates of each sensor node with respect to the base-station are randomly chosen using a uniform distribution of the angle in the $[0,2 \pi)$ interval, and a triangular distribution of the magnitude in the $(0, R$. interval.

Fig. 9 shows the value of the saturation throughput $(S)$ of the three retransmission protocols obtained using scenario A. Increasing values of energy per bit correspond to increasing values of the instantaneous signal to noise ratio which in terms lead to lower values of the error probability. When $E_{b_{s e n}}$ is high, the three protocols are able to achieve almost the same performance due to the low. error probability. Therefore, data frames have a high probability of being received correctly at the first transmission attempt and requiring no retransmissions. This is not optimal, as it does not take advantage of the collaborative nature of the protocols when retransmission of data frames is required. The three schemes are able to achieve the best value of the saturation throughput by finding the optimum in terms of tradeoff between the value of energy used to transmit each bit and the number of retransmissions necessary to ensure a reliable delivery of data frames. The ARQ-C and ARQ-C ${ }^{N}$ protocols reach saturation throughput values that may be more than twice the values achieved by the ARQ-NC protocol.

Figs. 10,11 , and 12 plot values of $S$ versus $E_{b_{s e n}}$, obtained using scenario $A$, for the ARQ-NC, ARC-C and ARQ-C ${ }^{N}$ protocol, respectively. A number of basestation RF antenna gains $(G)$ are considered. Intuitively, the value of $S$ grows with increasing values of $G$ for 


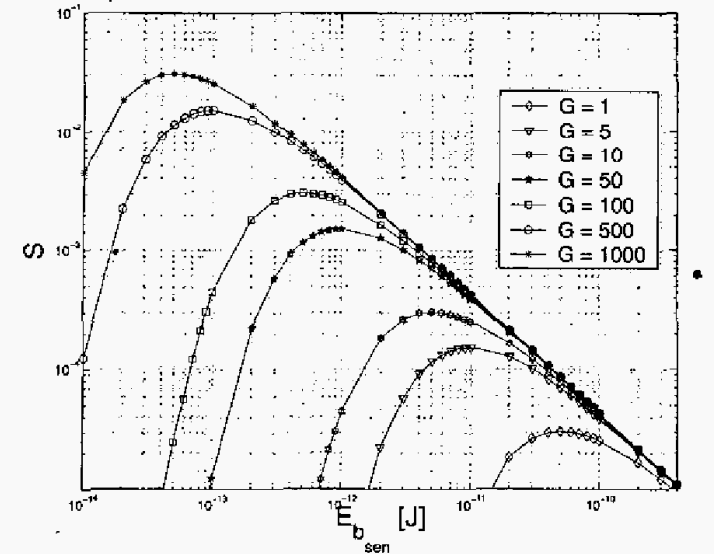

Fig. 10. Scenario A - ARQ-NC: saturation throughput ( $S$ ) versus energy per bit transmitted at the sensor nodes $\left(E_{b}\right)$. $R=50 \mathrm{~m}$, $n=3.5$

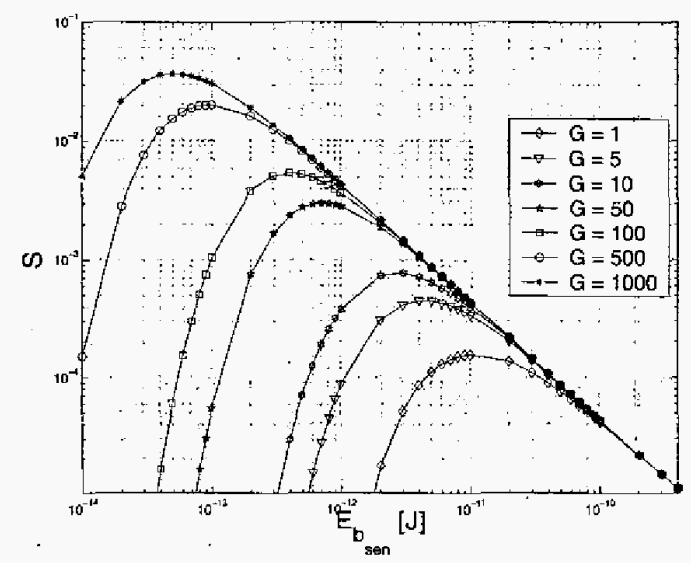

Fig. 11. Scenario A - ARQ-C: saturation throughput (S) versus energy per bit transmitted at the sensor nodes $\left(E_{b_{s e n}}\right) . R=50 \mathrm{~m}$, $n=3.5$

all of the three protocols. Cooperative communication is found to be more effective when the antenna gain is small, e.g., $G \leq 10$. The reason is that, while the antenna gain at the base-station increases, the RF antenna gain at the sensor node remains constant and equal to one, which favors transmission to the base-station when compared to transmission to cooperative sensor nodes.

Fig. 13 plots the value of $S$ as a function of the signal to noise ratio that is received at the base-station $\left(S N R_{B S}\right)$ obtained using scenario $\mathrm{B}$. In this case too, the two cooperative protocols may achieve higher values of $S$ when compared to the ARQ-NC protocol.

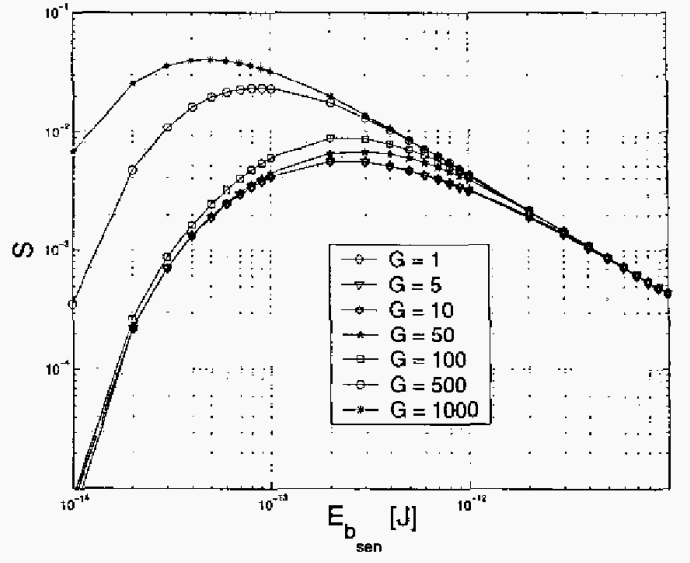

Fig. 12. Scenario A - ARQ-C ${ }^{N}$ : saturation throughput $(S)$ versus energy per bit transmitted at the sensor nodes $\left(E_{b_{s e n}}\right) . R=50 \mathrm{~m}$ $n=3.5$

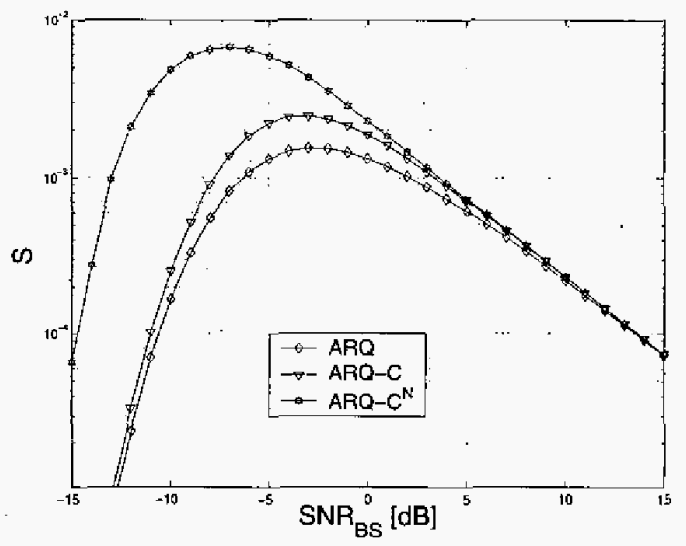

Fig. 13. Scenario B: saturation throughput $(S)$ versus signal to noise ratio received at the base-station $\left(S N R_{B S}\right), R=50 \mathrm{~m}, G=50$, $n=3.5$

The results in Table I document the effect of both the footprint radius $(R)$ and the path loss coefficient $(n)$ on $S$ for the three protocols when $G=50$. Results in the top part of the table are obtained using $E_{b_{s e n}}=1 \mathrm{E}-11 \mathrm{~J}$. Results in the top-left part are obtained using $n=3.5$. Results in the top-right part are obtained using $R=$ $50 \mathrm{~m}$. Results in the bottom part of the table are obtained using $S N R_{B S}=-5 \mathrm{~dB}$. Depending on the surrounding environment - which may affect the value of $n-$ the size of the footprint spans from tens to hundred meters. The two cooperative ARQ protocols yield always higher saturation throughput than the one achieved by the non cooperative ARQ. 
TABLE I

SATURATION THRQUGHPUT ( $S$ ) AS A FUNCTION OF THE FOOTPRINT RADIUS ( $R$ ) AND PATH LOSS COEFFCIENT ( $n$ )

\begin{tabular}{||c||c|c|c|c|c||c|c|c|c|c||}
\hline \multicolumn{1}{|c||}{} & \multicolumn{5}{c||}{$E_{u_{\text {sen }}}=1 \mathrm{E}-11 \mathrm{~J}, G=50, \mathrm{n}=3.5$} & \multicolumn{4}{c||}{$E_{b_{s e n}}=1 \mathrm{E}-11 \mathrm{~J}, G=50, R=50 \mathrm{~m}$} \\
\hline & $R=10 \mathrm{~m}$ & $R=20 \mathrm{~m}$ & $R=50 \mathrm{~m}$ & $R=80 \mathrm{~m}$ & $R=100 \mathrm{~m}$ & $n=2$ & $n=2.5$ & $n=3$ & $n=3.5$ & $n=4$ \\
\hline ARQ-NC & 0.121 & $1.07 \mathrm{E}-2$ & $3.90 \mathrm{E}-4$ & $4.83 \mathrm{E}-5$ & $1.19 \mathrm{E}-5$ & 154.7 & 2.183 & $3.07 \mathrm{E}-2$ & $3.90 \mathrm{E}-4$ & $3.31 \mathrm{E}-7$ \\
\hline ARQ-C & 0.121 & $1.07 \mathrm{E}-2$ & $4.31 \mathrm{E}-4$ & $7.26 \mathrm{E}-5$ & $2.31 \mathrm{E}-5$ & 154.7 & 2.183 & $3.08 \mathrm{E}-2$ & $4.31 \mathrm{E}-4$ & $1.40 \mathrm{E}-6$ \\
\hline ARQ-C & 0.121 & $1.07 \mathrm{E}-2$ & $4.34 \mathrm{E}-4$ & $8.29 \mathrm{E}-5$ & $3.35 \mathrm{E}-5$ & 154.7 & 2.183 & $3.08 \mathrm{E}-2$ & $4.34 \mathrm{E}-4$ & $4.45 \mathrm{E}-6$ \\
\hline & \multicolumn{9}{|c|}{$S N R_{B S}=-5 \mathrm{~dB}, G=50, \mathrm{n}=3.5$} \\
\hline & $R=10 \mathrm{~m}$ & $R=20 \mathrm{~m}$ & $R=50 \mathrm{~m}$ & $R=80 \mathrm{~m}$ & $R=100 \mathrm{~m}$ & $n=2$ & $n=2.5$ & $n=3$ & $n=3.5$ & $n=4$ \\
\hline ARQNC & 102.3 & 0.801 & $1.31 \mathrm{E}-3$ & $4.88 \mathrm{E}-5$ & $1.02 \mathrm{E}-5$ & $1.28 \mathrm{E} 7$ & $5.97 \mathrm{E} 3$ & 2.799 & $1.31 \mathrm{E}-3$ & $6.13 \mathrm{E}-7$ \\
\hline ARQ-C & 176.9 & 1.382 & $2.30 \mathrm{E}-3$ & $8.43 \mathrm{E}-5$ & $1.77 \mathrm{E}-5$ & $1.45 \mathrm{E} 7$ & $7.86 \mathrm{E} 3$ & 4.308 & $2.30 \mathrm{E}-3$ & $1.10 \mathrm{E}-6$ \\
\hline ARQ-C & 465.2 & 3.635 & $6.00 \mathrm{E}-3$ & $2.22 \mathrm{E}-4$ & $4.65 \mathrm{E}-5$ & $1.66 \mathrm{E} 7$ & $1.17 \mathrm{E} 4$ & 8.950 & $6.00 \mathrm{E}-3$ & $3.28 \mathrm{E}-6$ \\
\hline
\end{tabular}

\section{CONCLUSION}

The paper described the GAP4S architecture: a maintenance-free wireless sensor network. Three ARQ protocol options (two of which rely on cooperative communication) were proposed to yield reliable data delivery in GAP4S. The saturation throughput of the ARQ protocols was computed by solving a linear problem that characterizes the amount of cooperative communication offered by the sensor nodes.

In a variety of anticipated scenarios, it was found that the two ARQ protocols based on cooperative communication may more than double the saturation throughput, or equivalently, the required power to operate the system is half when compared to the non cooperative ARQ protocol. With acceptable microwave signal levels it is possible to reach footprint sizes in the hundred meter range. Possible fields of applications for GAP4S span from building, airport and monument monitoring and control, to industrial and agricultural activities, personal safety, monitoring and alerting systems.

Based on these encouraging results, further study is going to be carried out on cooperative ARQ protocols applied to sensor networks. For instance, it is interesting to investigate the transmission scheduling strategies at the base-station and the medium access control protocols that are best suited for GAP4S.

\section{ACKNOWLEDGMENT}

The authors would like to thank Isabella Cerutti, Stefano Gregori, Franco Maloberti, and Aria Nosratinia for the valuable technical conversations and input.

\section{REFERENCES}

[1] I. Akyildiz, W. Su, Y. Sankarasubramaniam, and E. Cayirci, "Wireless Sensor Networks: A Survey," Elsevier Computer Nerworks, wol. 38, no. 4, pp. 393-442, March 2002.

[2] D. Estrin, R. Govindan, J. Heidemann, and S. Kumar, "Next Century Challenges: Scalable Coordination in Sensor Networks," in Proceedings of Mobicom '99, August 1999, pp. 263 270.

[3] "Standard Wireless Protocols Create New Sensor Networks," National Aeronautics and Space Administration, Tech. Rep. 8 , August 2000 .

[4] D. Estrin, L. Girod, G. Pottie, and M. Srivastava, "Instrumenting the world with wireless sensor networks," in Proceedings of International Conference on Acoustics, Speech, and Signal Processing (ICASSP 2001), May 2001.

[5] J. Rabaey, M. Ammer, J. da Silva Jr., D. Patel, and S. Roundy, "PicoRadio supports ad hoc ultra-low power wireless networking," Computer, vol, 33, no. 7, pp. 42-48, 2000.

[6] W. R. Heinzelman, A. Chandrakasan, and H. Balakrishnan, "Energy Efficient Protocol for Wireless Microsensor Networks," in Proceedings of Hawail Intemational Conference on System Sciences, January 2000 , pp. 4-7.

[7] "http://dsn.east.isi.edu/."

[8] "http://lecs.cs.ucla.edu/galore."

[9] "htıp:/www.janet.ucla.edu/wins."

[10] "http://pads.east.isi.edu/."

[11] "http://bwrc.eecs.berkeley.edu/research/pico_radio/."

[12] "http:/www-mtl.mit_edu/research/icsystems/uamps."

[13] "http:/users.ece.gatech.edu/ weilian/sensor."

[14] "https:/www.fastlane.nsf.gov/servlet/showaward?award=0225528."

[15] A. Nosratinia, T. Hunter, and A. Hedayat, "Cooperative Communication in Wireless Networks," IEEE Communications Magazine, vol. 42, no. 10, pp. 74-80, October 2004.

[16] J. N. Laneman, G. W. Wornell, and D. N. C. Tse, "An efficient protocol for realizing cooperative diversity in wireless networks," in Proceedings of IEEE ISIT, June 2001, p. 294.

[17] M. Janani, A. Hedyat, T. Hunter, and A. Nosralinia, "Coded Cooperation in Wireless Communications: Space-Time Trans- 
mission and Iterative Decoding," IEEE Transactions on Signal Processing, vol. 52, no. 2, pp. 362-371, February 2004.

[18] A. Sendonaris, E. Erkip, and B. Aazhang, "User Cooperation Diversity-Part I: System Description," IEEE Transaction on Communications, pp. 1927-1938, November 2003.

[19] _ "User Cooperation Diversity-Part II: Implementation aspects and performance analysis," IEEE Transaction on Communications, pp. 1939-1948, November 2003.

[20] E. Zimmermann, P. Herhold, and G. Fettweis, "The impact of cooperation on diversity-exploiting protocols," in In Proceedings of 59th IEEE Vehicular Technology Conference (VTC Spring), May 2004.

[21] G. Zhang, T. Yang, S. Gregori, J. Liu, and F. Maloberti, "Ultralow Power Motion-triggered Image Sensor for Distributed Wireless Sensor Network," in Proceedings of the 2003 IEEE Intemational Conference on Sensors, October 2003, pp. 395 396.

[22] S. Gregori, Y. Li, H. Li, J. Liu, and F. Maloberti, " $245 \mathrm{GHz}$ Power and Data Transmission for a Low-Power Autonomous Sensors Platform," in the Proceedings of the 2004 International Symposium on Low Power Electronics and Design (ISLPED 2004), 2004, pp. 269-273.

[23] D. Bertsekas and R. Gallager, Data Networks (2nd ed.). Prentice-Hall, Inc, 1992.

124] J. Wall and J. Y. Khan, "An Advanced ARQ Mechanism for the 802.11 MAC Protocol," in Proceedings of Australian Telecommunications, Networks and Applications Conference(ATNAC), 2003.

[25] T. S. Rappaport, Wireless Communications; Principles and Practice (2nd Edition). Prentice Hall PTR, 2001.

[26] E. Malkamaki and H. Leib, "Evaluating the Performance of Convolutional Codes Over Block Fading Channels," IEEE Transactions on Information Theory, vol. 45, no. 5, pp. 16431646, July 1999.

[27] S. B. Wicker, Error Control Systems for Digital Communication and Storage. Prentice-Hall, 1995

[28] J. Hagenauer, "Rate-Compatible Punctured Convolutional Codes (RCPC Codes) and their Applications," IEEE Transaction on Communications, vol. 36, no. 4, pp. 389-400, April 1988.

[29] J. G. Proakis, Digital Communications (Fourth Edition). McGraw-Hill International Edition, 2001

[30] "http://www.ilog.com/products/cplex/." 